\title{
Efficacy of Probiotic Gargles in Reducing Post- operative Complications in Adult Post-tonsillectomy Patients: a Study Protocol for a Pilot Double-blinded, Randomised Controlled Trial and Feasibility Study
}

Michael Nasserallah ( $\nabla$ michael.nasserallah@gmail.com )

Monash University - Peninsula Campus https://orcid.org/0000-0003-3618-2785

Nalaka de Silva

Peninsula Health

Vicky Tobin

Peninsula Health

Warren Rozen

Peninsula Health

David Hunter-Smith

Peninsula Health

\section{Study protocol}

Keywords: Adenotonsillectomy, Tonsillectomy, Postoperative morbidity, Probiotics, Randomised controlled trial, feasibility study

Posted Date: April 19th, 2021

DOI: https://doi.org/10.21203/rs.3.rs-414380/v1

License: (c) (1) This work is licensed under a Creative Commons Attribution 4.0 International License.

Read Full License 


\section{Abstract}

Background: Tonsillectomy is one of the most commonly performed otolaryngologic procedures on children and adults. Despite improvements in surgical techniques, postoperative morbidity remains a significant clinical problem. Postoperative pain is the most common and incapacitating symptom that patients experience universally following tonsillectomy. For many years it has been suggested that posttonsillectomy pain is further worsened by colonisation of tonsillar fossae with organisms that induce an inflammatory response, thereby making pain worse. However, antibiotic treatment was not found to result in a clinically significant reduction in pain or postoperative analgesic requirements according to the most recent Cochrane review published in 2012.

Probiotics have shown to reduce inflammatory pain and improve functional abdominal pain in conditions such as irritable bowel syndrome. There are also several studies demonstrating that probiotics reduce recurrent pharyngotonsillitis, otitis media and other upper respiratory tract infections. The use of probiotics to manage post tonsillectomy pain is a novel idea that has not yet been investigated and may demonstrate promising results.

Aim: The aim of this pilot study is to determine the feasibility of a 14-day postoperative course of probiotic (Streptococcus salivarius K12) gargles compared to placebo gargles on morbidity following adenotonsillectomy in adults.

Design: This study is a double-blinded, randomised, placebo-controlled feasibility trial. The participants that will be recruited to this study are from the ENT waiting list at a single outer metropolitan hospital located in Melbourne, Australia.

Methods: Thirty adults, aged 18-55 years, all scheduled for elective tonsillectomy with or without adenoidectomy, will be included and assigned to receive either probiotic ( $S$. salivarius K12) gargles or placebo (isomalt) gargles for 14 days after surgery. The primary outcomes are postoperative pain and requirement of opiate analgesia. Secondary outcomes are assessing the safety and tolerability of probiotics and the feasibility of the trial. Study design will be assessed by any changes (during the pilot or recommended) to the protocol, recruitment by the numbers screened and time to enrol 30 participants; randomisation by the similarity of characteristics in groups at baseline, adherence will be assessed by the amount of probiotic/placebo gargle consumed, attendance to follow up appointments and completion of daily questionnaires; safety by will be examined by the number and description of adverse events and retention by the numbers withdrawn.

Discussion: This study will provide preliminary results that may support the feasibility of a large scale RCT to test the benefits of probiotic gargles post (adeno)tonsillectomy.

Trial registration: ACTRN 12619001474145; retrospectively registered 24/10/2019; First participant enrolled 14/10/2019; Australian and New Zealand Clinical Trial Registry 


\section{Introduction}

\subsection{Background and rationale $\{6 a\}$}

Tonsillectomy is one of the most commonly performed otolaryngologic procedures on children and adults. Despite improvements in surgical techniques, post-operative morbidity remains a significant clinical problem. Post-operative pain is the most common and incapacitating symptom that patients experience universally following tonsillectomy. Post-tonsillectomy pain can last up to two weeks and the resultant economic and social costs are considerable. This includes the inability to resume normal diet, a disrupted sleeping pattern, time off work and ultimately a reduced quality of life.(1) The requirement for additional analgesics, including opiates, is also associated with additional costs and potential complications such as gastrointestinal upset or respiratory depression. Furthermore, the incidence of postoperative haemorrhage varies from $2-40 \%$ and may incur additional morbidity in the form of readmission, blood transfusion and return to theatre for haemostasis.(2)

There are various theories behind the aetiology of postoperative pain in tonsillectomies, however it appears to be multifactorial. Nerve irritation, inflammation and pharyngeal muscle spasm have been proposed as the underlying mechanisms responsible for this complication of tonsillectomy. Therefore, anything that elicits any of these three factors will be a contributing factor to pain caused by tonsillectomy. The contributing components to post-tonsillectomy pain include: technical factors (such as choice of operative method), comorbidities (such as reflux), infection and dependent variables (such as age, gender and indication of surgery). (3)

The most widely proposed theory of post-tonsillectomy pain is the colonisation of the exposed tonsillar fossa with bacterial organisms, which induces a pro-inflammatory reaction and infection and contributes to post-operative morbidity. Hence, several authors recommend prophylactic antibiotics to reduce the morbidity.(4-6) Their routine perioperative administration had been well-established for decades in otolaryngology practice.

Despite earlier studies supporting the use of prophylactic antimicrobials in reducing post-operative pain, a 2012 Cochrane Review by Dhiwakar et al concluded that "there is no evidence to support a consistent, clinically important impact of antibiotics in reducing the main morbid outcomes following tonsillectomy (i.e. pain, need for analgesia and secondary haemorrhage rates)."(2) This review only included the use of systemic antibiotics as this practice is most commonly used postoperatively.

Nevertheless, there has been some literature to support the use of topical antibiotics post tonsillectomy to reduce pain. Topical administration of antibiotics has been effectively used for many years in colorectal surgery, with proven reduction in postoperative infections. Grandis et al (1994) subsequently showed the potential efficacy of topical antibiotic prophylaxis in preventing wound infections after contaminated head and neck surgery. Clindamycin, given as an oral rinse in this patient population, resulted in a striking reduction in overall oropharyngeal bacterial counts for at least 8 hours after the last dose and appeared to be more effective than parenteral clindamycin at reducing oropharyngeal bacterial counts.(7) However, 
despite previous studies demonstrating a reduction in postoperative morbidity in tonsillectomy patients with topical antibiotics,(4) the results from more recent trials have demonstrated no significant benefit from topical clindamycin.(8) It is important to note that most studies examining the effects of topical antibiotics post tonsillectomy have methodological flaws, such as small sample size and a significant proportion of participants lost to follow up, and thus further investigation is warranted.

Over the past thirty years, probiotics have emerged as a fascinating area in both health-related and commercial targets. In 2001, the World Health Organisation defined probiotics as "live microorganisms which when administered in adequate amounts confer health benefits on the host".(9) The role of beneficial bacteria on human health evolved from the work of Nobel Prize laureate Elie Metchnikoff in 1908. He investigated the longevity and general health of a population of peasants residing in Bulgaria that consumed primarily fermented dairy products and introduced the idea that lactic acid bacteria in yoghurt may counteract harmful gut pathogens and thus extend their life span.(10) It is now understood that the human body lives in a heavily contaminated bacterial environment, and symbiosis with these microorganisms seems to be a condition for survival. Furthermore, the human genome comprises of approximately 20,000 genes but the human microbiome (which includes the genes isolated from microorganisms that reside on or within human tissues and biofluids) constitutes 150 times more genes than that found in the human genome.(11) Hence, any disruption in the human microbiome, also known as a dysbiosis, may alter the normal function of that organ and result in disease. Therefore, a logical management approach to situations that alter our microbial ecology (e.g. diet, environment, antibiotics) would be to deliberately increase our association with specific non-pathogenic organisms to counter that alteration. Probiotics exert a wide spectrum of different effects ranging from direct antagonism against pathogens to influence upon intestinal epithelium and modulation of the immune system of the host's organism.(10)

Conventional probiotics have typically comprised of bacteria of intestinal origin (especially lactobacilli and bifidobacteria) and their application has principally been directed at managing gastrointestinal disorders such as inflammatory bowel disease, irritable bowel syndrome and diarrhoea. Furthermore, microbiome-directed therapies are increasingly used perioperatively and have been able to demonstrate a consistent reduction in postoperative complications in elective general surgery, upper gastrointestinal surgery and liver transplantation.(12) Under physiological conditions, the commensal intestinal bacteria induce tolerance responses and trigger the maintenance of immune homeostasis. Hence, probiotics have been theorised to prevent postoperative surgical site infections and morbidity by helping to restore the human microbiome.(13)

However, the realisation that much human illness can also be linked either directly (e.g. dental caries, periodontal disease and candidosis) or indirectly (e.g. cardiovascular disease and perhaps even obesity) to the development of oral microbiome dysbiosis has diverted much contemporary probiotic research to the development of products that are capable of fostering a healthy oral microbiome. While researchers initially tried to establish whether conventional approved intestinal probiotics could also influence the oral microbiome, these bacteria were unable to inhabit the oral mucosa and thus any oral cavity health 
benefits seem transitory and largely attributable to immune stimulation via action on the gastrointestinal tract.(14) Recently, studies have investigated the types of microbial communities that inhabit the upper respiratory tract. $(15,16)$ These studies show quite a difference between the key bacterial players in the respiratory tract compared to the ones in the gastrointestinal tract. Therefore, a more logical strategy is to utilize microbes isolated from their natural oral habitat in healthy humans as oral probiotics.

Streptococcus salivarius is already established in the human oral cavity a few hours after birth and remains there as a predominant commensal inhabitant. Streptococcus salivarius $K 12$ has been shown to have inhibitory activity against pathogenic organisms known to cause upper respiratory tract infections and periodontitis. Additionally, S. salivarius has been found to affect immune responses by inhibiting inflammatory pathways activated by the pathogens, suggesting a role in the modulation of human epithelial cell immune responses.(17)

In 2001, the K12 strain became the first $S$. salivarius to be commercially developed as a probiotic and more than 50 million doses have now been marketed internationally by the New Zealand company BLIS Technologies Ltd (Dunedin, New Zealand).

The S. salivarius K12 strain produces two lantibiotic bacteriocins Salivaricin A2 and Salivaricin B, also known as bacteriocin-like inhibitory substances (BLIS). This bacteriocins can effectively counteract the growth of beta-haemolytic (group A) Streptococcus pyogenes, which is a common cause of pharyngitis, tonsillitis, and acute otitis media. $(17,18)$

Additionally, the K12 strain can also inhibit the growth of Haemophilus influenzae, Streptococcus pneumoniae, Moraxella catarrhalis, Micrococcus luteus, Streptococcus anginosus, Eubacterium saburreum, and Micromonas micros. Many of these are potential pathogens in the ear and oral cavity, causing pharyngotonsillitis, acute otitis media and halitosis. (17)

Furthermore, in vitro and in vivo studies have demonstrated that the $\mathrm{K} 12$ strain also has immunomodulatory properties and contributes actively to the host defense process by downregulating an inflammatory response by inhibiting the NF-kB pathway, interfering with IL-8 synthesis, and suppressing IL-8 secretion.(19)

The safety of strain K12 has been specifically supported by a series of studies affirming the absence of known virulence factors and antibiotic resistance determinants, its low mutagenicity predisposition, acute and subacute toxicity testing in rats, and a successful high-dosage trial in humans.(20-22) The consensus from these strain-specific studies, has enabled a self-affirmed 'generally regard as safe (GRAS)' status to be granted for strain K12 in the USA.(21) Moreover, the double-blinded randomised, placebo-controlled study reported by Burton et al suggests that the daily ingestion of high-dose $S$. salivarius K12 ( $1 \times 10^{\wedge} 10$ colony forming units) over a 28-day period does not adversely affect the human host, that it is safe and well tolerated as an oral probiotic.(21) 
The aim of this study is to evaluate the efficacy of probiotic (S. salivarius K12) gargles in adult posttonsillectomy patients in reducing postoperative complications, primarily, post-operative pain. There are no similar studies to date as yet available in the medical literature. This study will be a double-blinded, randomised controlled pilot study that will take place over an 18-month period with adult participants who will have (adeno)tonsillectomies at Frankston Public Hospital. We will investigate the effect of 14 days of probiotic gargles post-tonsillectomy and whether this reduces pain compared to placebo gargles.

If probiotics can reduce post-operative complications in adult tonsillectomy patients, we could reduce morbidity, reduce requirement for opiate analgesia, prevent overuse of antibiotics and reduce substantial costs to the healthcare system.

\subsection{Objectives $\{7\}$}

The primary objective of this study is to compare the postoperative pain and morbidity following elective (adeno)tonsillectomy in adults treated with postoperative probiotic (S. salivarius K12) or placebo (isomalt) gargles at Peninsula Health.

The secondary objective of this study is to compare the safety and adverse effect profile of probiotic gargles compared to placebo gargles. The third objective of this trial, is to determine whether this study is feasible by examining the study design, the ability to recruit and screen participants, the randomisation process, adherence to trial protocols, safety of the trial intervention and the retention of participants.

We hypothesise that probiotics post tonsillectomy will help reduce postoperative pain and morbidity and will have less adverse effects compared with placebo. We also hypothesise that this trial is feasible.

\subsection{Trial design \{8\}}

This study is single-centre, double-blind, randomised controlled trial and feasibility study. Patients will be randomly allocated to receive either probiotic (Streptococcus salivarius K12) gargles or placebo (isomalt) gargles for 14 days after surgery.

\section{Methods}

\subsection{Study setting $\{9\}$}

This trial will be conducted at a single-site, Frankston Public Hospital, and the participants will be adult patients undergoing elective tonsillectomy with or without adenoidectomy.

\subsection{Eligibility criteria $\{10\}$}

Participants will be assigned to a randomised trial treatment only if they meet all of the inclusion criteria and none of the exclusion criteria. 


\subsubsection{Inclusion criteria}

1. Age 18-55

2. Indication of operation (one of the following): asymmetric tonsillar enlargement, recurrent tonsillitis, previous quinsy (> one), sleep disordered breathing, halitosis, tonsillolith

3. Elective Tonsillectomy or adenotonsillectomy only

4. Weight must be more than $50 \mathrm{~kg}$

\subsubsection{Exclusion criteria}

1. Allergic to opioids / codeine / Endone ${ }^{\mathrm{TM}}$ / paracetamol

2. Lactose intolerance

3. Unstable metabolic diseases/disorder

4. Heart failure or a history of endocarditis

5. Immunocompromised

6. Kidney disease

7. Liver disease

8. Haemorrhagic diathesis

9. Other concomitant surgical procedures

10. Pregnancy

11. Patients who are currently taking NSAIDs e.g.: Celebrex ${ }^{\mathrm{TM}}$, Aspirin, etc.

12. Patients who are on regular opiates or Paracetamol

13. Recent use of probiotics within 1 month

\subsection{Informed consent process $\{26 \mathrm{a}\}$}

Prior to performing any trial-specific procedure, a signed consent form will be obtained for each participant. Participants will be adult patients (see Eligibility criteria) who are a placed on the Peninsula Health waiting list awaiting (adeno)tonsillectomy. Participants will be sent a letter of invitation (see Appendix 1) and patient information/consent form (PICF) (see Appendix 2) informing them that they will be contacted by the investigator regarding the willingness to participate in the trial. Participants who do not wish to be contacted will contact the waiting list and advise they do not wish to be contacted by the associate investigator. One week after the participant has received the letter of invitation and PICF they will be contacted by the associate investigator and discuss the trial with the participant.

The PICF document will describe the purpose of the trial, the procedures to be followed, and the risks and benefits of participation. The investigator will meet with participants 1 week prior to surgery to ensure that they meet the eligibility criteria, answer any questions they may have, explain the trial in further detail 
and check that the participant comprehends the information provided. The participant will have a chance to discuss with family and friends before the day of surgery. If a patient wishes to participate in the trial, they will be invited to provide written consent. Consent will be voluntary and free from coercion. The investigator who conducted the consent discussion will also sign as witness on the informed consent form. A copy of the signed consent form will be given to the participant on discharge. It will be documented in the participant's record that consent has been provided. When all the eligibility criteria have been addressed and confirmed, the pharmacist will be informed they are a participant and will assign the patient a study ID number and to a trial arm/intervention. Participants screened that are ineligible for participation in trial will be recorded in the electronic database with reason of ineligibility, age, sex and indication of surgery.

\subsection{Additional consent provisions for collection and use of participant data and biological specimens $\{26 \mathrm{~b}\}$}

Not applicable, as no samples will be collected.

\section{INTERVENTIONS}

\subsection{Choice of Comparator $\{6 \mathrm{~b}\}$}

The control group will be using placebo gargles composed of isomalt powder as the primary ingredient. The rationale behind the selection of isomalt powder was that it does not cause any harm and has almost identical physical and chemical properties as the probiotic powder used in this trial. Both powders are white in colour and are identical in appearance and taste to the probiotic powder. Furthermore, if the probiotic group did receive benefit, the designers of the study wanted to distinguish benefit from the actions of the probiotic organism and benefit from the act of gargling itself.

\subsection{Intervention description $\{11 a\}$}

Eligible patients will be randomised in equal proportions between probiotic and placebo.

\subsubsection{Treatment arms}

All patients will receive paracetamol, endone, prednisolone, metoclopramide, coloxyl and senna and Movicol in a general pack.

The trial arm will receive $S$. salivarius $K 12$ powder and the placebo arm will receive isomalt powder. 


\subsubsection{Trial Intervention(s)}

All medications are administered through the oral route

- Paracetamol 1g QID

- Oxycodone 5mg, PRN/4Hrly

- Prednisolone 25mg BD for 3 days

- Metoclopramide 10mg TDS prn for nausea/vomiting

- Coloxyl and senna 1-2 tabs BD for constipation

- Movicol 1-2 sachets BD for constipation

- Streptococcus salivarius K12 or placebo powder - Dissolve 2 scoops of powder in $20 \mathrm{mls}$ of warm water and gargle for 30 seconds then swallow. Perform this 4 times a day, for 14 days ideally after brushing teeth and before meals, starting 24 hours post surgery

\subsubsection{Storage, preparation, dispensing and administration of trial drug}

The trial medication and placebo control will be purchased from an independent Community pharmacy and the Red Spoon Company respectively. The powders will be packaged by the trial pharmacist from the Department of Pharmacy at Peninsula Health, who will prepare the trial packs. On day 1 posttonsillectomy surgery the trial pack will be given to participants in identical medicinal containers with a unique ID number allocated to each participant placed on the label.

The labels we will place on the medication will also include the following instructions: "For clinical trial use only", "Keep out of reach of children", "Dissolve 2 scoops of powder in $20 \mathrm{ml}$ of warm water and gargle for 30 seconds then swallow. Perform this 4 times a day, ideally after brushing teeth and before meals. Continue treatment for 14 days" and "store below 25 deg" along with any local or national requirements.

\subsubsection{Modifications}

No dose modifications are required.

\subsubsection{Surgery and postoperative management}

\section{Surgery}

All patients will be operated on by a single experienced surgeon carried out under general anaesthesia with the use of orotracheal intubation. The post nasal space is inspected and an adenoidectomy is performed if clinically necessary, using curettage or with suction diathermy device at $25 \mathrm{~W}$ (using a Valleylab Force FX' Electrosurgical Generator C). The post nasal space is then packed with 1:10,000 
adrenaline soaked raytec gauze. The tonsils are then removed using a standardised method of electrodissection, with monopolar electrocautery set at $15 \mathrm{~W}$. Haemostasis will be secured with bipolar cautery set at $12 \mathrm{~W}$. A local analgesic will then be topically applied using 0.75 per cent ropivacaine soaked paediatric raytec gauze.

\section{Postoperative management}

After surgery, patients will be transferred to the post-anaesthesia care unit for monitoring, and then moved to the ward once they have awoken and deemed safe. During the postoperative period, patients will receive regular analgesics, with allowance for IV morphine or oral endone for breakthrough pain relief, ondansetron and metoclopramide as antiemetics, and other medications as deemed necessary. The remaining two doses of $8 \mathrm{mg}$ of IV dexamethasone will be administered on the ward 8 hours apart.

\subsubsection{Follow-up}

\section{Follow-up}

On the day of discharge, the participants will be reviewed by the ENT team and postoperative instructions will be provided. These instructions will explain how to use the trial medications, analgesia and all other medications prescribed. Furthermore, participants will be advised to consume regular rough textured foods, to coordinate the consumption of analgesia approximately 30-60 minutes prior to meals and to avoid any strenuous activity for 2 weeks after surgery. After review by the ENT team, the trial pharmacist will then meet with participants and provide the trial medication and other medications prescribed and also explain the correct method of using the trial powder.

Participants will be sent an electronic survey (Daily 14-day questionnaire) to their mobile or email daily via SurveyMonkey ${ }^{\circledR}$ daily (see Appendix 3). Alternatively, a paper form of the diary (see Appendix 4) will be provided to the participant if they do not have access to mobile or email.

A telephone review on day 5, using a preformed script (See Appendix 5), will be organised to ensure that participants are managing their pain adequately, correctly performing the gargles and to ensure there are no serious adverse effects. On day 14, participants will be reviewed by the associate investigator in the outpatient clinic to examine the wound bed and the general health status of the patient. Participants will be advised to return any unused powder from the trial intervention, which will be delivered to the trial pharmacist and weighed as a measure of compliance. On day 28, participants will be reviewed by the study investigator in the outpatient clinic to examine the wound bed and the general health status of the patient. If a participant requires additional information or analgesia, they are advised to speak to the study investigator at any time from day 1 to day 28 post surgery. The entire process from patient allocation to follow-up is displayed in Figure. 1 and further described in a SPIRIT (Standard Protocol Items: Recommendations for Intervention Trials) figure in Figure 2. 


\subsubsection{Covid-19 precautions}

Due to COVID-19 restrictions on trial continuity and trial protocols the following amendments will be made.

- Day 5 follow-up will be via telephone as per protocol

- Day 14 and Day 28 follow-ups will be in the outpatient clinic as per protocol.

- Prior to the outpatient visit the associate investigator will contact patients and ask if they have any fevers or respiratory symptoms, close contact with COVID suspected/proven patients or if they have tested positive for COVID

- If a patient has no risk factors, they will be seen in the outpatient clinic as planned. The associate investigator will wear gloves, mask and eye protection during the consultation and conduct appropriate hand hygiene. The patient will also be advised to wear a mask and conduct appropriate hand hygiene.

- At the end of the consultation a disinfectant wipe will be used to wipe down all surfaces contacted by the patient and himself and then appropriate hand hygiene will be completed.

- If the patient has any of the above risk factors, the associate investigator will advise them to get a COVID swab test if they have not had one already and to remain self-isolated until the result is negative and then see them in the outpatient clinic. If this is not feasible a telephone consult will be completed using the same questions from Day 5 telephone consult.

- If participants are not able to attend the outpatient clinic, they will be advised to bring pack the remaining powder to the pharmacy at day 14 as per protocol. If participants are unable to return unused medication themselves a family member will be asked to do so. Powder containers will be handled with gloves and appropriate hand hygiene when returning it to the trial pharmacist.

\subsection{Adherence $\{11 \mathrm{c}\}$}

Completion of the patient diary specifically designed for this study will help monitor if participants are adherent to the treatment regime. This electronic survey form is sent to each participant's mobile or email daily via SurveyMonkey®.

Participants who do not complete the online patient diary questionnaire will be notified and prompted to complete the questionnaire by text message from associate investigator, Michael Nasserallah.

Furthermore, at the 14th day visit post surgery, participants will be advised to return any unused drugs, which will be collected by the investigator and given to the pharmacist to measure the amount of powder remaining. The trial pharmacist will maintain accurate records of all trial medication, including dates of receipt. In addition, accurate records will be kept regarding when and how much trial medication is dispensed and used by each participant in the trial. At the end of the trial, there will be final reconciliation 
of trial drug received, dispensed, consumed and returned. Any discrepancies will be investigated, resolved and documented by the trial team.

\subsection{Concomitant care $\{11 \mathrm{~d}\}$}

\section{Concomitant therapy}

On induction of general anaesthesia patients will receive a dose of $1.2 \mathrm{~g}$ of IV benzylpenicillin (or $600 \mathrm{mg}$ of IV Clindamycin if allergic) once only to reduce risk of bacteraemia and to eliminate the presence of pathogenic organisms.(23) A single dose of parecoxib 40mg IV will be administered by the anaesthetist intraoperatively to help reduce pain and the need for rescue analgesia in the immediate postoperative period.(24) Perioperatively patients will receive 3 doses of $8 \mathrm{mg}$ IV dexamethasone 8 hourly to reduce pain and improve patient's recovery in immediate postoperative period.(25) The first dose will be given on induction of general anaesthesia.

\section{Excluded medications and treatments}

NSAIDs are not to be used for duration of trial medication use (i.e., 2 weeks). Antibiotics should not be used unless patients are admitted with severe pain, dehydration or secondary haemorrhage with clinical evidence of infection. If antibiotics are prescribed by the general practitioner then the participant will be withdrawn from trial treatment.

See Exclusion criteria for full list of excluded medications.

\subsection{Ancillary and post-trial care $\{30\}$}

Patients that are enrolled into the study are covered by Medicines Australia Standard Form of Indemnity. For full details regarding compensation guidelines please visit the following link: https://medicinesaustralia.com.au/wp-content/uploads/sites/52/2010/09/CInical-Trials-CompensationGuidelines-1.pdf.

Peninsula health is also insured to cover for non-negligent harm that may reach participants associated with this protocol.

Should this study provide evidence of the feasibility and safety of probiotic gargles in adult patients post tonsillectomy, we will aim to conduct a powered study. A research grant will be applied for in order to conduct additional data collection in the form of swab results and blood tests in order to elucidate the mechanism by which probiotics can reduce pain.

\subsection{Flow chart of trial process}




\subsection{Participant timeline $\{13\}$}

\subsection{Treatment discontinuation, participant withdrawals and losses to follow up $\{11 \mathrm{~b}\}$}

Participant withdrawals and losses to follow up will be documented clearly.

\subsubsection{Discontinuation of treatment - participant remains in trial for follow up}

Participants will be required to document how often they used the probiotic or placebo gargle in their daily pain diary. Participants will be asked to bring any unused powder with them on the day 14 visit to measure how much of the trial drug or placebo remains unused in order to estimate compliance.

Participants may discontinue trial treatment for the following reasons:

- Participant requests to discontinue trial intervention

- Investigator decision to discontinue a participant from the trial intervention if the participant:

- Is pregnant

- Demonstrates significant non-compliance with the trial intervention

- Experiences a serious or intolerable adverse event such that continued trial intervention would not be in the best interest of the participant

- Develops, during the course of the trial, symptoms or conditions listed in the exclusion criteria

- Requires a medication that is prohibited by the protocol

- Requires early discontinuation for any other reason

The investigator may also withdraw all trial participants from the trial treatment if the trial is terminated. For the safety of all participants ceasing trial treatment, the protocol-specified safety evaluations should be undertaken to capture new safety events and to assess existing, unresolved safety events. All scheduled follow-ups of trial participants should also occur following treatment discontinuation, where possible. A dedicated Case Report Form (CRF) page will capture the date and the specific underlying reason for discontinuation of the trial intervention. The participant should remain in the trial for scheduled visits for trial assessments (follow-up) per protocol.

\subsubsection{Withdrawal of consent - participant withdraws from all trial participation}


Participants are free to withdraw from the trial at any time upon their request. Withdrawing from the trial will not affect their access to standard treatment or their relationship with the hospital and affiliated health care professionals.

For the safety of all participants ceasing trial treatment, reasonable efforts should be made to undertake protocol-specified safety evaluations to capture new safety events and to assess existing, unresolved safety events following withdrawal.

The last page on the PICF "Form for Withdrawal of Participation" will be used to capture the date and reason of participant withdrawal of consent. Participant withdrawal from the trial should only occur if the participant withdraws their consent to continue any trial involvement. This can occur at any stage of the trial following consent, including prior to receiving the intervention, while receiving the intervention or during the follow up phase.

\subsubsection{Losses to follow-up}

A participant will be considered lost to follow-up if he or she fails to return for either day 14 or day 28 scheduled visits and is unable to be contacted by the trial site staff. The following actions will be taken if a participant fails to return to the clinic for a required trial visit:

- The associate investigator will attempt to contact the participant and reschedule a meeting within 23 days of the missed visit and counsel the participant on the importance of maintaining the assigned visit schedule and ascertain if the participant wishes to and/or should continue in the trial.

- Before a participant is deemed lost to follow-up, the associate investigator or designee will make every effort to regain contact with the participant (where possible, up to 3 telephone calls and, if necessary, a certified letter to the participant's last known mailing address or local equivalent methods). These contact attempts will be documented in the participant's medical record or trial file.

- Should the participant continue to be unreachable, he or she will be considered to have withdrawn from the trial with a primary reason of lost to follow-up.

\subsubsection{Replacements}

Participants who sign the informed consent form but are not randomised or assigned to a trial intervention may be replaced. Participants who have been randomised or assigned to a trial intervention may be replaced. If a patient consents to participate in the trial, receives the trial medication but is then lost to follow up, recruitment of an additional person can be arranged via notifying pharmacy to give the same treatment. The investigator and participant would remain blinded until the end of study.

\subsubsection{Trial closure}


A participant is considered to have completed the trial if he or she has completed all phases of the trial including the last visit or the last scheduled procedure shown in Figure. 1. The end of the trial is defined as completion of the last visit in the trial for all participants. At this stage, the associate investigator will ensure that all HRECs and RGOs as well as all regulatory and funding bodies have been notified.

This trial may be temporarily suspended or prematurely terminated if there is sufficient reasonable cause. If the trial is prematurely terminated or suspended, the associate investigator will promptly inform trial participants, HREC and RGO, the funding and regulatory bodies, providing the reason(s) for the termination or suspension. Circumstances that may warrant termination or suspension include, but are not limited to:

- If the bleeding rate requiring surgery or blood transfusion is greater than 1 in 10 participants, or readmission for pain or dehydration is greater than 1 in 10 participants or the participant has postoperative sepsis within 2 weeks post-surgery that is not explainable by any other cause

- Determination of an unexpected, significant, or unacceptable risk to participants that meets the definition of a Significant Safety Issue (SSI) (for the definition refer to Section 3.16 Safety/harms).

- Insufficient compliance to protocol requirements

- Data that are not sufficiently complete and/or evaluable

- Demonstration of efficacy that would warrant stopping

- Determination that the primary endpoint has been met

- Determination of futility

In the case of concerns about safety, protocol compliance or data quality, the trial may resume once the concerns have been addressed to the satisfaction of the HREC, RGO, funding and/or regulatory bodies.

\subsection{Outcomes $\{12\}$}

\section{Outcomes}

\section{Primary outcome}

The primary outcome is post (adeno)tonsillectomy pain and the requirement of opiate analgesia. Participants will be sent an electronic survey to their mobile or email daily via SurveyMonkey ${ }^{\circledR}$. Alternatively, a paper form of the diary will be provided to the participant if they do not have access to mobile or email. Daily pain scores will be determined by the participant diary specifically designed for this study (Daily 14-day questionnaire).

The average daily pain scores at rest, drinking and eating over the preceding 24-hour period, using a numerical rating scale ( 0 being no pain to 10 being the worst pain experienced), will be recorded by the participant. These scores will be recorded daily for 14 days post surgery. Additionally, the daily requirement of paracetamol and opiate analgesics will be recorded in number of tablet(s) consumed. 
That is the number of $500 \mathrm{mg}$ tablets of paracetamol and the number of $5 \mathrm{mg}$ tablets of oxycodone (ENDONE) the participant consumed over a 24-hour period.

\section{Secondary outcome}

The secondary outcomes assessing the safety and tolerability of probiotics include:

- The readmission rate due to dehydration or poor oral intake as measured by hospital records.

- The amount of side effects daily experienced (e.g. nausea, vomiting, constipation, drowsiness, halitosis, abdominal pain) will be documented by participants in the diary specifically designed for this study.

- The rate of post-tonsillectomy haemorrhage as assessed by hospital records using a 4-point Haemorrhage scale ( 1 = no bleeding; $2=$ minimal bleeding less than a mouthful, managed at home with ice gargles; 3 = moderate bleeding, managed medically managed in hospital with gargle/silver nitrate cautery without blood transfusion requirement; 4 = Profuse bleeding, requiring theatre or needed blood transfusion).

\section{Feasibility measures}

\section{Design}

- Study design will be assessed by examining the ability and time required of study staff to coordinate recruitment, screening and clinic tasks. The ability of staff to contact participants, the duration of initial phone call to participants and the staff required for initial recruitment phone call.

- The screening and clinic tasks will also be assessed by recording the number of staff required and the duration (in minutes) of interviews at participant visits on day 5 (via phone), day 14 and day 28 (in outpatient clinic).

\section{Recruitment and screening}

- Will be assessed by the time required and number of people screened to enrol 30 participants to completion of final trial visit

\section{Randomisation}

- Will be determined by ability to achieve balance of characteristics in each group

\section{Adherence}

- Adherence in both groups will be assessed by amount of probiotic/placebo gargle consumed as measured by participant diaries and bottle return, attendance at follow up appointments and completion of daily questionnaires 
- Safety will be assessed by the number and description of both serious adverse events (any admission to the emergency department or hospitalisation, life threatening events or results in significant morbidity or death) and other adverse events by group. Serious adverse events (including haemorrhage requiring operative intervention or blood transfusion, readmission due to pain and dehydration or septicaemia) will be adjudicated by an Independent Medical Monitoring team (two independent Ear Nose and Throat specialists) as either unrelated, possibly related, probably related, or definitely related to study.

\section{Retention}

- Retention will be assessed by the number of participants that withdrew by group.

\subsection{Sample size $\{14\}$}

As this trial is a feasibility pilot study, no prior power estimations were performed and no tests of efficacy or statistical significance will be provided for the between group differences in both primary and secondary outcomes. However, a $95 \%$ confidence interval will be provided to give information about the magnitude and direction of an effect. As in visual analogue scales, a change on the numerical rating scale of $20 \%$ between two time-points of an assessment is regarded as being clinically significant.(26)

We have elected to choose a total number of 30 patients to be included in the trial ( 15 patients per group). This number was chosen as we are limited by the number of patients currently on the waiting list awaiting tonsillectomy or adenotonsillectomy. We have also elected this number in order to demonstrate the safety and feasibility of this trial. Outcome measures such pain, use of analgesics, and rates of readmission will be calculated and a statistical analysis will be performed. We will use the results from this study to perform a more accurate power analysis in designing a larger clinical trial.

\subsection{Recruitment $\{15\}$}

We aim to accrue a total of 30 patients, which will likely take 6-12 months to complete. Information regarding patients (potential participants) awaiting tonsillectomy or adenotonsillectomy surgery will be provided by the waiting list department at the Frankston Hospital, Peninsula health. Patients on the waiting list who meet the eligibility criteria will be sent a letter of introduction (see Appendix 1), the patient information and consent form (PICF) (see Appendix 2) along with the pre-operative information pack by the waiting list department at Peninsula Health. This should reach the patients 2 to 3 weeks before they are scheduled for their surgery, allowing them time to read, reflect and consult with family and or friends. The introductory letter and the PICF outline the study in detail. Participants will then be contacted 1-2 weeks prior to surgery, by the associate investigator to ask if they have received the preoperative information pack, if they have any questions and their willingness to participate. If a patient is willing to participate in the study, they will be invited to meet with the associate investigator prior to surgery in order 
to explain the surgery and the trial in more detail and provide an opportunity for patients to clarify the information they have received.

On the day of surgery, patients will have been already consented for tonsillectomy or adenotonsillectomy surgery prior by the ENT consultant. If participants have notified the researcher of their willingness to participate, the study investigator can then further explain and answer any questions about the study. To confirm consent to participate in the trial, the patient will be asked to sign a copy of the PICF they had received prior to surgery. A signed copy of the PICF will be provided to the patient upon discharge. The recruitment process will stop after 30 participants have completed the study treatments and data collection for all endpoints.

\section{ALLOCATION}

\subsection{Sequence generation $\{16 a\}$}

The trial pharmacist(s) who will not be involved in the collection or analysis of the trial results will prepare the randomisation schedule using block randomisation to maintain balance between treatment arms. The schedule will be provided by the pharmacist who will also provide the treatment allocation of each randomisation code in a sealed envelope to the study principle investigator in case of emergency.

\subsection{Concealment mechanism $\{16 \mathrm{~b}\}$}

A computer-generated randomisation (using Microsoft Excel ${ }^{T M}$ ) is undertaken by the Department of Pharmacy. The trial or placebo pack will be provided in a sealed plastic bag to the participant and will be recorded on a password protected electronic database and in the blinded section of the pharmacy trial folder.

\subsection{Implementation $\{16 \mathrm{c}\}$}

A patient will be identified as eligible to participate in the trial by the study investigator, who will then provide the patient details to the trial pharmacist(s). A list of randomly assigned treatments produced by the pharmacist, which allocates treatment to participants in chronological order will be formulated prior to commencement of study. The study investigator will then provide the names of participants to the pharmacist as they are recruited. For example, the first participant is study ID\#1, they will receive treatment pack for study ID\#1 by the pharmacist.

On postoperative day 1 the pharmacist will then deliver the medication to the participant prior to discharge. The medication will have an ID number and the pharmacist will be the only person who knows whether the medication is the probiotic or the placebo. The pharmacist will have the participant details, 
what trial group they belong to and what item number of the medication the patient has received. This information will be stored in the blinded section of the pharmacy clinical trial folder, accessible to the pharmacist alone. The pharmacist will only inform the statistician which treatment each participant (identified by study ID number) has received after the completion of all data collection. Thus, randomisation will be conducted without any influence of the principal investigators, associate investigators or statistician.

\section{BLINDING}

\subsection{Blinding mechanism \{17a\}}

This trial is a double-blinded study and as described above, the associate investigator will not have access to what trial product patients have received. Additionally, the appearance and taste of the probiotic and placebo powders are almost identical and, as this is not a cross over design, patients will receive one only type of treatment, so be unable to compare appearance or taste of the two treatments. Therefore, trial participants will also remain blinded for the duration of the study. The daily 14-day questionnaire will ask patients to answer what ID number of the trial pack they have received in order to ensure that patients have received the assigned treatment at the conclusion of the study.

At the conclusion of the study, the pharmacist conducting the randomisation and allocation of trial treatments will be notified. The pharmacist will be asked to split up the two groups into Group A and Group B patients. The pharmacist will then provide the list of the two groups to the statistician without revealing which group received the probiotic and which received the placebo. This additional method will help remove any potential bias during the statistical analysis.

\subsection{Emergency unblinding $\{17 b\}$}

No breaking of the trial blind is required unless the bleeding rate requiring surgery or blood transfusion is greater than 1 in 10 participants, or readmission for pain or dehydration is greater than 1 in 10 participants or the participant has postoperative sepsis within 2 weeks post-surgery that is not explainable by any other cause.

The randomisation code for an individual participant may only be unblinded in emergency situations, where the Investigator decides a participant cannot be adequately treated without knowing the identity of their treatment allocation. To break the randomisation code the investigator must open the emergency unblinding envelopes provided, or contact the trial pharmacist who performed the randomisation schedule. If any emergency unblinding occurs, the time, date, participant number and reason for unblinding must be documented. 


\section{DATA COLLECTION AND MANAGEMENT}

\subsection{Trial procedures and evaluations $\{18 \mathrm{a}\}$}

The daily post-tonsillectomy questionnaire is adapted from a pain diary used in previous published study with the author's permission.(27) Pain diaries are a reliable and validated method of assessing pain.(27) The numerical rating scale used in this pain diary was selected over other pain scales as it has shown high correlations with other pain-assessment tools in several studies. The feasibility of its use and good compliance have also been proven it is a reliable and validated method of pain measurement.(26)

All data will be collected by the study investigator in electronic form via the Daily 14-day questionnaire specifically designed for this study. This questionnaire is designed to be sent automatically via SurveyMonkey ${ }^{\circledR}$ to the patient's email or mobile each day at $6 \mathrm{pm}$ and participants are advised to provide answers reflecting a 24-hour period (i.e., $7 \mathrm{pm}$ the night before to $7 \mathrm{pm}$ the current day). If a trial participant has not completed the survey for that day then the associate investigator will receive a notification and a text reminder will be sent to the participant to complete the survey.

If the patient decides to complete the paper form of the diary instead, this will be collected by the associate investigator at the 14-day review in the outpatient clinic. Furthermore, data will be collected from the hospital records for up to 28 days post surgery for each trial participant regarding readmission and presentations to the emergency department.

Each trial participant will be given a trial pack (which will contain the probiotic or placebo) and be allocated a number by the pharmacist packaging the medication. The pharmacist will be the only person who has information of which patient is receiving what product until the completion of data collection. Patient pain diaries (via electronic questionnaire or hard copy) will be collected by the associate investigator. While data collection and data entry into spreadsheets for data analysis continues, this data will be identifiable by patient UR numbers to ensure there is no data duplication, and data entry is accurate. Data must remain identifiable until the completion of data collection and the pharmacist provides the treatment schedule of each participant, identified by their name and UR number. Data for each participant will also be identified by a unique study ID number.

Once data collection is complete, the pharmacist will be notified to provide a list of the two separate treatment groups by their ID numbers without revealing which group is the intervention and which is the placebo. The excel spreadsheet data will be de-identified by the deletion of each participant's name and UR number and henceforth identified only by the study ID number.

All data will be stored on a password-protected Microsoft excel spreadsheet and saved on the secure Peninsula Health servers, which are also password protected. This data will be accessible by all team members directly involved in the research project, i.e. the principal investigator and associate investigators. All hard copy data or information collected will be scanned and stored on to the same 
computer mentioned above and then disposed of in a secure information bin at the completion of data collection and entry into the excel spreadsheet. After all data has been collected for all 30 patients, the statistician, will have access to this de-identified data to perform the statistical analysis. As mentioned previously, the analysis will be performed by the statistician on two groups of patients, without the knowledge of which group was allocated to probiotic and which group was allocated to placebo.

\subsection{Retention $\{18 b\}$}

Once a patient is enrolled or randomised, the investigator will make every reasonable effort to follow the participant for the entire study period. Participants who do not complete the online patient diary questionnaire will be notified and prompted to complete the questionnaire by text message from the associate investigator. Furthermore, participants will be encouraged to contact the associate investigator directly if they have any questions regarding their postoperative care which will promote the retention of trial participants.

\subsection{Data management $\{19\}$}

\section{Source Data}

The "Daily 14-day questionnaire" is an electronic survey, which will also be available in paper form, will be the primary means in which data is collected. The survey is sent to participants via SurveyMonkey ${ }^{\circledR}$ and thus all data points are received by the investigators electronically. The account linked to this survey portal is password protected and only accessible by the associate investigator. The questionnaire that is sent daily for 14 days post surgery is identical apart from having a unique collector ID associated with each day post surgery. For example, postoperative Day 1 has the collector ID 01, Day 2 post operatively has collector ID 02 etc.

At the completion of the trial, i.e. when all 30 participants have completed their day 28 postoperative follow up appointment, the data from SurveyMonkey ${ }^{\circledR}$ will be inputted into a secured Microsoft excel spreadsheet stored on the Hospital server and all data Identifiers will then be removed. The completed excel spreadsheet will be password protected and accessible only to the investigators of this study. All data on paper files will also be de-identified and inputted into the Microsoft excel spreadsheet. The paper files will also be scanned and kept in a locked filing cabinet in the Department of Surgery. All electronic files will be kept on the hospital server which is password protected.

\section{Record retention}

At the completion of the trial, all the data will be stored on the hospital computer server and on a Monash university LabArchives Electronic Lab Notebook account, accessible by the investigators, for at least 5 years following the publication of the trial results. 


\subsection{Confidentiality $\{27\}$}

All study-related information will be stored on the Peninsula Health hospital computer server in password protected documents. All information regarding consent and trial visits will be stored on the Hospital computer server and will be accessible to clinicians who provide direct care to participants. All data from Daily 14-day questionnaire will be initially stored on SurveyMonkey ${ }^{\circledR}$ and will be only accessible by the associate investigator, Doctor Michael Nasserallah. At the conclusion of the study, i.e. after the $30^{\text {th }}$ patient has completed the final trial visit, the data stored on SurveyMonkey ${ }^{\circledR}$ will be extracted and placed into a Microsoft excel spreadsheet. The data will be de-identified and participants will only be known by patient ID number alone. This data spreadsheet will be password protected and accessible by only the trial pharmacist and statistician performing the analysis. The pharmacist conducting the randomisation and allocation of trial treatments will be notified. The pharmacist will split up the two groups into Group A and Group B patients. They will provide the list of the two groups, identifiable by study ID alone, to the statistician without revealing which group received the probiotic and which received the placebo. This additional method will help remove any potential bias from the statistical analysis.

\subsection{Plans for collection, laboratory evaluation and storage of biological specimens for genetic or molecular analysis in this trial/future use $\{33\}$}

Not applicable, no samples collected.

\section{STATISTICAL METHODS}

\subsection{Outcomes $\{20 \mathrm{a}\}$}

The collected data will be entered into a Microsoft Excel spreadsheet. Baseline characteristics that will be presented include date of birth, sex, indication of surgery, date of surgery and surgery type (tonsillectomy versus adenotonsillectomy).

Primary outcome:

- Post (adeno)tonsillectomy pain and the requirement of opiate analgesia.

These will be assessed by calculating: mean or median pain scores at rest, and when eating or drinking, for each postoperative day (days 1 to 14); number of Endone tablets taken (per day and in total); first postoperative pain-free day. These will be compared between the two treatment arms.

Secondary outcome: 
- Assessing the safety and tolerability of probiotics.

We will calculate the number of participants who experienced nausea, vomiting, diarrhoea, constipation, bleeding or drowsiness; number of side effects incidents per day; numbers and types of side effects encountered postoperatively; and incidence of post-tonsillectomy haemorrhage. The bleeding profiles will be assessed using a four-point haemorrhagic scale. These will be compared between the two treatment arms.

Feasibility measures:

- Is the trial feasible?

Study design will be assessed by any changes (during the pilot or recommended) to the protocol, recruitment by the numbers screened and time to enrol 30 participants; randomisation by the similarity of characteristics in groups at baseline, adherence will be assessed by amount of probiotic/placebo gargle consumed, attendance to follow up appointments and completion of daily questionnaires; safety by number and description of adverse events and retention by numbers withdrawn.

\section{Statistical method used}

The distribution of continuous data will be determined by Shapiro Wilks test for normality as either normal or not normal. Comparisons will use appropriate tests for the assessed distribution: t-tests or ANOVAs (for normal data) or Wilcoxon-Mann Whitney or Kruskal Wallis (for non-normal data) depending on nature of data and its distribution. The data may also be assessed by regression analysis including generalized linear mixed models (GLMM). The appropriate models will be used when applied to normal and non-normal data. GLMM are especially useful when data structure is complex and not independent e.g. comparison of multiple data points for each participant to be compared across treatment arms. These regression analyses will also allow us to test for effect of modifiers such as whether the surgery indicators and or surgery type influence the outcome/s. Categorical data will be presented as percentage frequency and assessed using Pearson's chi-square or when a contingency table contains a population of less than five, Fisher's exact test will be used. We may also use these in appropriate regression analyses such as logistic regression. $P$ values $\leq 0.05$ will be accepted as being statistically significant. All statistical analyses will be performed using Stata IC version 16 (StataCorp, College Station, Texas, USA).

\section{Presentation of data}

Data will be presented either in tables or graphs. Normally distributed continuous data will be described as mean \pm standard deviation and non-normally distributed data as median \& interquartile range in either bar or box graphs or in tables. Categorical data will be described as percent frequency \& samples size and presented as either bar graphs or in tables.

Comparisons: 
The output of regression or generalised linear mixed model analysis will be described as either regression co-efficient or odds ratios, and $95 \%$ confidence intervals. The output of t-tests and ANOVAs and their equivalents for non-normally distributed data will be reported as the output of the test (ie t or $Z$ value) and the $p$-value. Where appropriate, post-hoc analysis will be conducted. For example when assessing time course of reported pain at rest, eating or drinking over days following surgery will be assessed for overall significance and if found, post hoc analysis using eg Holm-Sidak test which compares all pairs against a control group (placebo, and or time - eg pre tonsillectomy or Day 1 post-tonsillectomy, depending on data). Only two-sided statistical tests will be used and reported. The numbers of missing data will be assessed and reported.

\subsection{Interim analysis $\{21 b\}$}

An interim analysis will not be performed unless clear evidence of benefit or harm is demonstrable in one group versus the other. However, due to the limited numbers in this pilot trial the performance of an interim analysis is unlikely to be performed.

\subsection{Additional analyses $\{20 \mathrm{~b}\}$}

If there is a difference or variability in the adherence of participants in taking their gargles, we may explore if there is a correlation between the number of gargles used to outcomes. For example, is there a relationship between the number of gargles used and the amount of endone required over the 14 days OR is there a relationship between the number of gargles used and pain scores.

\subsection{Analysis population and missing data $\{20 \mathrm{c}\}$}

\section{Population to be analysed}

We will be analysing the intention to treat population as defined below.

Intention to treat population (ITT): Includes any participant randomised into the trial, regardless of whether they received trial drug.

\section{Handling of missing data}

A participant is non-adherent if they fail to attend at least 1 of the 2 follow up visits (i.e. Day 14 or Day 28). Participants will be contacted if medications are not collected by Day 28 to allow for complete analysis of results. Participants will also be contacted daily if they do not complete the online Daily 14day questionnaire. 


\subsection{Plans to give access to the full protocol, participant level data and statistical code $\{31 \mathrm{c}\}$}

The protocol, fully study report, de-identified data set and statistical code for generating results remain the intellectual property of Monash University and will be available at the conclusion of the Masters of Surgery Thesis in October 2021.

\section{OVERSIGHT AND MONITORING}

\subsection{Composition of the coordinating centre and trial steering committee $\{5 d\}$}

\section{Trial Management Group (TMG)}

The Site Principal Investigator is responsible for supervising any individual or party to whom they have delegated tasks at the trial site. They must provide continuous supervision and documentation of their oversight. To meet this Good Clinical Practice (GCP) requirement, a small group will be responsible for the day-to-day management of the trial and will include at a minimum the Site PI and project manager/research nurse/trial coordinator. The group will closely review all aspects of the conduct and progress of the trial, ensuring that there is a forum for identifying and addressing issues. Meetings must be minuted with attendees listed, pertinent emails retained and phone calls documented.

\section{Trial Steering Committee (TSC)}

A TSC will be established to provide expert advice and overall supervision, and ensure that the trial is conducted to the required standards. The TSC will meet at least monthly, with more frequent meetings as needed, and will work to a Terms of Reference. The TSC will comprise of all authors listed above under the heading "Protocol Contributors". Michael Nasserallah will be responsible for identification, recruitment, data collection and completion of case report forms (CRFs), along with follow up of study patients and adherence to study protocol.

\section{Data manager}

Sole responsibility of associate investigator Michael Nasserallah. This will include ensuring data is collected and stored appropriately.

\section{Independent Medical Monitoring team}

Independent medical monitor team, comprising of two ENT consultants (Mr Diamanti Diamantaras and Mr Vibhuti Mahanta), together with the investigator will assess the seriousness of adverse events and the relationship of the event to the trial intervention. 


\subsection{Composition of the data monitoring committee, its role and reporting structure $\{21$ a $\}$}

Data monitoring is the sole responsibility of the associate investigator. A data monitoring committee was not formed due to the short duration of the trial and the assessed minimal risks of this trial.

\subsection{Safety/harms $\{22\}$}

The following section was adapted from the Murdoch Children's Research Institute "Clinical Trial (Drug) Protocol" template.(28)

\subsubsection{Definitions for use in trials involving investigational medicinal products}

\section{Participant-specific adverse events}

Adverse Event (AE): Any untoward medical occurrence in a patient or clinical trial participant administered a medicinal product and does not necessarily have a causal relationship with this treatment.

Adverse Reaction (AR): Any untoward and unintended response to an investigational medicinal product related to any dose administered.

Comment: All adverse events judged by either the reporting investigator or the sponsor as having a reasonable possibility of a causal relationship to an investigational medicinal product would qualify as adverse reactions. The expression 'reasonable causal relationship' means to convey, in general, that there is evidence or argument to suggest a causal relationship.

Serious Adverse Event (SAE)/ Serious Adverse Reaction (SAR): Any adverse event/adverse reaction that results in death, is life threatening, requires hospitalisation or prolongation of existing hospitalisation, results in persistent or significant disability or incapacity or is a congenital anomaly or birth defect.

Note: Life-threatening refers to an event in which the participant was at risk of death at the time of the event. It does not refer to an event that hypothetically might have caused death if it were more severe.

Medical and scientific judgement should be exercised in deciding whether an adverse event/reaction should be classified as serious in other situations. Important medical events that are not immediately lifethreatening or do not result in death or hospitalisation but may jeopardise the participant or may require intervention to prevent one of the other outcomes listed in this definition should also be considered serious. 
Suspected Unexpected Serious Adverse Reaction (USAR): An adverse reaction that is both serious and unexpected.

Consider a SUSAR as any SAE that is both suspected to be related to the trial treatment and is unexpected (i.e. not consistent with the available safety information in the Investigator's Brochure (for unapproved products) /approved Product Information or device labelling (for approved products). Note that SUSARs require expedited reporting to stakeholders including the Sponsor, Investigators, HREC, local governance office and TGA.

Safety issues (requiring expedited reporting). (see Appendix 6 for examples of SSIs).

\section{Events requiring expedited reporting}

The following definitions describe additional safety events that require expedited reporting to stakeholders including the Sponsor, Investigators, HREC, local governance office and TGA.

Significant Safety Issue (SSI): A safety issue that could adversely affect the safety of participants or materially impact on the continued ethical acceptability or conduct of the trial. Comment: A SSI is a new safety issue or validated signal considered by the principal or associate investigators in relation to the investigational medicinal product that requires urgent attention of stakeholders. This may be because of the seriousness and potential impact on the benefit-risk balance of the investigational medicinal product, which could prompt regulatory action and/or changes to the overall conduct of the clinical trial, including the monitoring of safety and/or the administration of the investigational medicinal product.

Urgent Safety Measure (USM): A measure required to be taken in order to eliminate an immediate hazard to a participant's health or safety. Note: This is a type of SSI that can be instigated by either the principal or associate investigators and can be implemented before seeking approval from HRECs or institutions.

\section{Safety monitoring}

Safety monitoring will be conducted solely by the associate researcher Dr Michael Nasserallah who will collaborate with the Independent Medical Monitoring team regarding adverse events or outcomes encountered and report them to HREC and TGA as appropriate. Any safety events will be reported as per Peninsula Health policy "Monitoring and Reporting of Safety in Clinical Trials Involving Therapeutic Goods and Other Clinical Research". Examples of significant safety issues (SSI) that require expedited reporting are listed in appendix 6.

The study reported by Burton JP et al (23) assessed the safety and potential impact on health parameters, oral and intestinal tolerance of human subjects to $S$. salivarius K12. In brief, this study found:

- The participants' perception was that regardless of whether they were taking the probiotic or placebo there was no significant change in the condition of their teeth, the freshness of their breath or the extent of gum bleeding at the end of either the treatment phase or wash out phase when compared 
to their baseline values $(P>0.05)$. They did report a significant difference in tooth sensitivity, which was reduced for the probiotic group when compared to the placebo group at the end of the treatment period

- The results for abdominal pain complaints, bloating, gastric reflux, nausea, vomiting, diarrhoea and flatulence were not significantly different between the two treatment groups at the various assessment points throughout the study $(P>0.05)$. There was however, a significant difference $(P=$ 0.006 ) between the probiotic and placebo groups after the treatment period because those in the placebo group felt that their general gastrointestinal health had declined over the period, whereas the average response for those in the probiotic group had remained relatively stable.

- There are no SAEs reported with the use of $S$. salivarius K12 compared to placebo (Figure 2) (23). The reported AEs in the article by Burton JP et al such as mild abdominal pain, mild changes in appetite, mild infection, all did not require treatment; moderate headache occurred in one out of 27 patients and required simple analgesics. (23)

\subsubsection{Capturing and eliciting adverse event/reaction information}

Adverse events and adverse reactions (non-serious and serious) will be captured from the time of administration of the investigational medicinal product until 14 days after the final dose is taken and will be followed until resolution or stabilisation. At every trial visit, participants will be asked "How have you felt since your last visit?" in order to elicit any medically related changes in their well-being. They will also be asked if they have been hospitalised, had any accidents, used any new medication or changed concomitant medication regimens. In addition, AEs will be documented from physical examination findings, clinically significant lab results or other documents (including participant diaries and correspondence from their primary care physician) that are relevant to participant safety.

\subsubsection{Documentations of AEs}

For the purposes of this trial the associate investigator is responsible for recording all Adverse Events, regardless of their relationship to trial drug, with the following exceptions:

- Conditions that are present at screening and do not deteriorate will not be considered adverse events.

- Abnormal laboratory values will not be considered adverse events unless deemed clinically significant by the investigator and documented as such.

The AE will be described in participants medical record and captured on the dedicated CRF and will include:

- A description of the $\mathrm{AE}$

- The onset date, duration, date of resolution

- Severity (mild, moderate or severe - what is the impact on the participant's daily life?) 
- Seriousness (i.e. is it a SAE?)

- Any action taken, (e.g. treatment, follow-up tests)

- The outcome (recovery, death, continuing, worsening)

- The likelihood of the relationship of the AE to the trial treatment (Unrelated, Possible, Probable, Definite) as determined by the Independent Medical monitoring team

Changes in the severity of an AE will be reported. AEs characterised as intermittent will be documented for each episode. All AEs will be followed to adequate resolution, where possible.

\subsubsection{Assessing the seriousness of participant's AE}

According to the definitions for all clinical trials hospitalisation due to progression of disease will not be considered a SAE for the purposes of this trial. The severity and relationship of an AE will be assessed as per the following section. The seriousness of an AE will be assessed by an investigator in conjunction with the Independent Medical Monitory team according to the NHMRC definition for SAE.(29)

\subsubsection{Assessing the relatedness (causality) of a participant's $A E$}

All serious adverse events must have their relationship to trial intervention assessed by the Independent Medical monitoring team who will evaluate the adverse event based on temporal relationship and his/her clinical judgment. The degree of certainty about causality will be graded using the categories below. In a clinical trial, the trial product should always be suspected.

The relationship of the event to the trial intervention will be assessed as follows:

- Unrelated: There is no association between the trial intervention and the reported event. AEs in this category do not have a reasonable temporal relationship to exposure to the test product, or can be explained by a commonly occurring alternative aetiology.

- Possible: The event could have cause or contributed to the AE. The AEs in this category follow a reasonable temporal sequence from the time of exposure to the intervention and/or follow a known response pattern to the test article, but could also have been produced by other factors.

- Probable: The association of the event with the trial intervention seems likely. AEs in this category follow a reasonable temporal sequence from the time of exposure to the test product and are consistent with the known pharmacological action of the drug, known or previously reported adverse reactions to the drug or class of drugs, or judgement based on the investigators clinical experience.

- Definite: The AE is a consequence of administration of the trial intervention. AEs in the category cannot be explained by concurrent illness, progression of disease state or concurrent medication reaction. Such events may be widely documented as having an association with the test product or that they occur after rechallenge. 


\subsubsection{Assessing the severity and expectedness of a participant's $A E$}

The severity of an Adverse Event will be assessed as follows:

- Mild: Events that require minimal or no treatment and do not interfere with the participant's daily activities.

- Moderate: Events that cause sufficient discomfort to interfere with daily activity and/or require a simple dose of medication.

- Severe: Events that prevent usual daily activity or require complex treatment.

The associate investigator will be responsible for determining whether an adverse event (AE) is expected or unexpected. An AE will be considered unexpected if the nature, severity, or frequency of the event is not consistent with the risk information previously described for the trial intervention.

\subsubsection{Reporting of safety events}

Site Principal Investigator Reporting Procedures

The Site Principal Investigator is responsible for recording all safety events in the source document. The associate investigator is responsible for expedited reporting (within 24 hours of becoming aware of the event) to the principal investigator the following local safety events:

1. USMS

2. SUSARS

3. All SAEs /SARs, except those that are identified below as expected in the trial population:

- Increasing pain at day 5-7 post-operatively is an expected progression; self-resolving, low volume haemorrhage not requiring operative management can occur anytime between Day 4 - day 10; postoperative nausea and vomiting is a common complication.

The associate Investigator is responsible for reporting SAEs (including SUSARs) to the principal investigator as soon as possible but within 24 hours of the first knowledge of the event. These reports should be submitted using the trial Expedited Safety Report Form (see Appendix 7).

The Site Principal Investigator is responsible for reporting SSIs, local USMs and local SUSARs to their research governance office within 72 hours of becoming aware of the event and in accordance with their local governance authorisation.

\section{Associate Investigator Reporting Procedures}

The associate investigator must assess and categorise the Expedited Safety Reports recorded and report these to the Principal Investigator, the approving HREC and TGA in accordance with the NHMRC's 'Safety 
monitoring and reporting in clinical trials involving therapeutic goods'(29) and any additional requirements of the approving HREC. All safety reports must clarify the impact of the safety event on participant safety, trial conduct and trial documentation. The associate investigator is responsible for the following reporting to the Principal Investigator, the HREC and TGA:

1. All SSIs that meet the definition of a USM within 72 hours of becoming aware of the issue.

2. All other SSIs within 15 calendar days of instigating or becoming aware of the issue

3. For SSIs leading to an amendment of trial documentation:

4. For SSIs leading to temporary halt or early termination of a trial for safety reasons:

The Principal investigator will also report SUSARs to the TGA as follows:

1. Fatal or life-threatening SUSARs immediately, but no later than 7 calendar days after being made aware of the issue (follow up info within a further 8 calendar days.

2. All other SUSARs no later than 15 calendar days of being made aware of the issue

The Principal investigator is responsible for providing the additional safety information to the approving HREC:

1. Provide an annual safety report, including a summary of the evolving safety profile of the trial

2. Provide any updated Product Information/Investigator's Brochure for the investigational products (if applicable)

The Principal investigator is also responsible for providing any updated Product Information/Investigator's Brochure to Investigators.

\subsection{Auditing}

\subsubsection{Site Monitoring \{23\}}

Trial site monitoring is conducted to ensure that the rights and well-being of trial participants are protected, that the reported trial data are accurate, complete, and verifiable, and that the conduct of the trial is in compliance with the currently approved protocol and amendment(s), good clinical practice and applicable regulatory requirements.

Full details of trial site monitoring are documented in the Clinical Monitoring Plan (CMP) below. The CMP describes in detail who will conduct the monitoring, at what frequency monitoring will be done, at what level of detail monitoring will be performed, and the distribution of monitoring reports. 
Monitoring for this trial will be performed by the associate investigator. On-site, weekly monitoring for the first month of the trial will be conducted which will involve review of $100 \%$ of original signed consent forms, trial eligibility data and data related to primary outcome, safety and other key data variables. A review of all withdrawals from trial treatment and/or trial follow up and a targeted review of other data including investigational medicinal product administration and accountability.

\subsubsection{Quality Control and Quality Assurance $\{23\}$}

The Principal investigator has responsibility in relation to quality management. The Principal and associate investigators will develop Standard Operating Procedures (SOPs) that identify, evaluate and control risk for all aspects of the trial, e.g. trial design, source data management, training, eligibility, informed consent and adverse event reporting. The associate investigator will also implement quality control (QC) procedures, which will include the data entry system and data QC checks. Any missing data or data anomalies will be communicated to the site for clarification/resolution.

As outlined in the previous section (Site Monitoring), the trial monitor will verify that the clinical trial is conducted and data are generated, documented (recorded), and reported in compliance with the protocol, good clinical practice and applicable regulatory requirements. In the event of non-compliance that significantly affects human participant protection or reliability of results, the principal and associate investigators will perform a root cause analysis and corrective and preventive action plan (CAPA).

\section{DISSEMINATION PLANS}

\subsection{Dissemination policy \{31a\}}

The author of this protocol (Michael Nasserallah) holds the primary responsibility of the results of the trial and Monash University will have intellectual property over the results. Participants will be notified at the completion of the trial via SMS or email and offered the chance to receive a report of the overall results. Individual results will not be reported as data analysis will be only with de-identified data. The participants will receive a letter of thanks which will also let them know whether they were part of the treatment or placebo arm.

The results of this study will form part of the Masters of Surgery thesis for Dr Michael Nasserallah. We will also aim to present these results in annual meetings at Peninsula health such as Celebrating Research, Department of Surgery Surgical Symposium and monthly Department of Surgery research meetings. Furthermore, the results of this study will be presented at national and or international medical conferences and we will also aim to publish the results of this study in a peer-reviewed journal.

\section{Discussion}


The idea for this trial was proposed by Mr Nalaka de Silva who provided anecdotal evidence for the use of probiotic gargles post tonsillectomy to reduce pain. The research team discussed the feasibility of this project and proposed a randomised controlled trial. The associate investigator, Dr Michael Nasserallah, discussed with Maria Stolaki (Winclove Probiotics Research Institute) and Prof John Tagg and Prof John Hale (lead researchers from BLIS Technologies Ltd) in order to seek professional advice from researchers who had been involved in the study of probiotics. Winclove Probiotics supply a powder which contains the probiotic strains, Streptococcus oralis 89a and Lactobacillus rhamnosus $21 \mathrm{~b}$. BLIS technologies supply a single probiotic strain, Streptococcus salivarius K12, which is available in powder and lozenge form. The decision to use Streptococcus salivarius K12 was made due to the large amount of literature available for its support as an anti-inflammatory and prevention against pathogenic organisms known to cause upper respiratory tract infections. Furthermore, the $S$. salivarius K12 is listed on the Australian Register of Therapeutic Goods (ARTG) as an approved substance for oral health, and this strain is also available commercially in community pharmacies in Australia.

\section{COVID-19 impact on trial}

Due to the COVID-19 pandemic, this clinical trial incurred a delay in recruitment and follow up of patients. Due to restrictions on elective operations, the proposed time required to recruit all 30 patients is expected to increase from 6 months to 18 months. Furthermore, an adjustment to outpatient visits was made in order to maintain infection control standards see section 5.2.7.

\section{TRIAL STATUS}

\subsection{Protocol amendments}

The following amendments were made: 


\section{Version \\ Number \\ Summary of changes \\ and Date}

$1 ; 23.01 .19$ Original

2; 13.03.19 Changed pilot study to test feasibility and investigate the possible treatment effect;

Removed blood tests (serology markers) - no available funding

Removed saliva samples for quantitative PCR - no available funding or laboratory to store samples

Removed halimeter readings - equipment unreliable and too expensive

3; 07.09.19 Formatting of protocol

Change of stakeholders and funding

Initially the probiotic and placebo products were going to be supplied and provided by BLIS technologies Ltd but we were unable to come to an agreement regarding intellectual property.

Therefore, the associate investigator, Michael Nasserallah, was able to source probiotic and placebo commercially.

Funding was provided by the Academic Unit, Department of Medicine, Central clinical school, Peninsula Health and by the Peninsula Health Department of Surgery.

4

17.09.2019

Formatting of protocol

Response to Human research ethic committee outcome

- Reframing trial as feasibility study

- Confirmation of recruitment method including a flow chart and provision of telephone script

- Appointment of Independent Medical monitor to review adverse events

- Clarification of pain endpoints

- Provision of both a paper and electronic patient diary

- Revision of post tonsillectomy diary as Celebrex mentioned

- Patient information consent form formatting

- Submission of Clinical trials notification via Therapeutic Good Australia (TGA)

- Registration for ANZCTR commenced

- Clarification of ARTG status of probiotic

5 ;

20.07 .2020

Adjustment to trial visit protocols due to COVID-19 


\subsection{Recruitment date commenced and expected completion}

This trial is in the recruitment phase. The first participant was enrolled in the trial on October 14, 2019. The expected time to trial completion was 6-12 months post trial registration. However, given the restrictions on elective operating due to COIVD-19 pandemic the trial is expected to conclude recruiting in 18 months from trial registration date (October 24, 2019).

\section{Abbreviations}




\begin{tabular}{|c|c|}
\hline 4hrly & 4 hourly \\
\hline $\mathrm{AE}$ & Adverse Event \\
\hline ANOVA & Analysis of Variance \\
\hline AR & Adverse Reaction \\
\hline $\mathrm{BD}$ & Twice a day \\
\hline CRF / eCRF & Case Report Form / electronic Case Report Form \\
\hline DMC SMC & Data Monitoring Committee / Safety Monitoring Committee \\
\hline DSMB & Data Safety Monitoring Board \\
\hline FDA & Food and Drug Administration \\
\hline GCP & Good Clinical Practice \\
\hline GLP & Good Laboratory Practices \\
\hline GMP & Good Manufacturing Practices \\
\hline HREC & Human Research Ethics Committee \\
\hline IB & Investigator's Brochure \\
\hline IMP & Investigational Medicinal Product \\
\hline ITT & Intention To Treat \\
\hline IV & Intravenous \\
\hline MCRI & Murdoch Children's Research Institute \\
\hline NHMRC & National Health and Medical Research Council \\
\hline NSAIDs & Non-steroidal Anti-inflammatory Drugs \\
\hline OD & Once daily \\
\hline $\mathrm{PI}$ & Product Information (available for an approved drug or device) \\
\hline $\mathrm{PI} / \mathrm{CPI}$ & Principal Investigator / Coordinating or Chief Principal Investigator \\
\hline PICF & Participant Information Sheet/Consent Form \\
\hline PO & Orally \\
\hline PRN & When necessary \\
\hline QA & Quality Assurance \\
\hline QC & Research Governance Office \\
\hline QID & Four times a day \\
\hline
\end{tabular}




\begin{tabular}{|ll|}
\hline SAE & Serious Adverse Event \\
\hline SAR & Serious Adverse Reaction \\
\hline SMC & Safety Monitoring Committee \\
\hline SOP & Schedule of Assessments \\
\hline SSI & Standard Operating Procedure \\
\hline SUSAR & Significant Safety Issue \\
\hline TDS & Suspected Unexpected Serious Adverse Reaction \\
\hline TGA & Three times a day \\
\hline UAR & Unerapeutic Goods Administration \\
\hline USM & Urgent Safety Measure \\
\hline
\end{tabular}

\section{Declarations}

\subsection{Authorship \{31b\}}

The order of authorship will be as follows: MN, NDS, VT, WR, DHS.

Contributions to development of the study protocol, literature review, ethics application, overall conducting the trial, analysis of data and write up of results was and will be performed by MN.

Contributions to the theoretical concept of the study, trial design and medical expertise was performed by NDS.

Contributions to the review of the study protocol, ethics application, data analysis and interpretation of results was and will be performed by VT.

Contributions to the study design, conduct, review of submitted drafts, interpretation of study results and overall supervision of trial was performed by WR.

Contributions to the study design, conduct, review of submitted drafts, interpretation of study results and the principal supervision of trial was performed by DHS.

All authors read and approved the final manuscript.

\subsection{Acknowledgements}

The following acknowledgements will be made to: 
Ms Janet Thompson from the Peninsula Health Library for assisting in the literature review search.

Ms Lee-Anne Clavarino, Manager Office for Research at Peninsula Health for overall guidance and support of the trial.

Clinical trials Pharmacists at Peninsula Health for conducting the randomisation process of the trial, packaging, labelling and dispensing the trial products and overall support of the trial.

Professor Srikanth Valendai and the Peninsula Clinical School, Central Clinical School, Monash University for funding and overall support of the trial.

The Department of Surgery, Peninsula Health for funding and overall support of the trial.

Professor John Tagg and Professor John Hale, lead researchers from BLIS Technologies Ltd, for assisting in discussions regarding the feasibility of the study design and for providing advice regarding the appropriate dose and method of probiotic to utilise in this study.

Ms Maria Stolaki, from Winclove Probiotics Research Institute, for assisting in discussions regarding the selection of the appropriate probiotic strain and for overall support of the trial.

The Murdoch Children's Research Institute (MCRI) Clinical Research Development Office (CRDO) for allowing the use of the "Clinical Trial (Drug/Device) Protocol, annotated" template for this publication.

The Australian Government for its support through the Research Training Program (RTP) Scholarship.

\subsection{Funding $\{4\}$}

We have no trial sponsor. We will be receiving funding from Peninsula Health's research department to supply the probiotic and placebo samples but no monies. Furthermore, we will receive funding from the Peninsula Clinical School, Central Clinical School, Monash University to cover the fees incurred by Department of Pharmacy to conduct randomisation, packaging and dispensing of probiotic and placebo. Both sources of funding have no role in the design of the study, analysis, interpretation or writing of this manuscript.

\subsubsection{Stakeholder involvement}

BLIS technologies Ltd are the principal manufacturers of the probiotic used in this study. The associate investigator has collaborated with Prof John Tagg and Prof John Hale. We have discussed the appropriateness and feasibility of the research study. We also discussed the use of high-dose probiotic powder for the trial and methods to optimise the clinical study. 
We will purchase the probiotic sample and placebo treatment for each trial participant. The probiotic "Blis Probiotics Daily Defence Junior" will be purchased from a community pharmacy and the placebo isomalt powder will be purchased from the Red Spoon Company (an online company that sell modernist cuisine ingredients and cookware). Both products will be packaged in identical medicinal bottles by the Department of Pharmacy at Peninsula Health and will be dispensed to the patient. During the explanation of trial information and consenting procedure, each trial participant will be provided with a full list of ingredients of both products, irrespective of which treatment they ultimately receive. If they are allergic to any ingredient for either product they will be excluded from the study.

\subsection{Availability of data and materials $\{29\}$}

All principal investigators, the trial pharmacist and statistician will be given access to the de-identified data. Furthermore, the Independent Medical Monitoring team will also be given access to the de-identified data sets and provided with the opportunity to reassess SAE and their relationship to the trial intervention. The data that supports the findings of this study will be available from Monash University but restrictions apply to the availability of these data and thus will not be publicly available. However, data will be available from the authors upon reasonable request and with permission of Monash University.

\subsection{Research ethics approval and consent to participate $\{24\}$}

This protocol, PICF, patient instructions and diary sheet, telephone script prior to surgery, letter of invitation to participate, telephone script for day 5 review, Victorian specific module (VSM), Clinical Trials notification (CTN) and site-specific approval was reviewed by the Human Research and Ethics Committee (HREC) at Peninsula Health. Ethics approval (Reference: HREC/51745/PH-2019) was granted on the $17^{\text {th }}$ of September 2019, please see appendix 8. Furthermore, the study will be conducted according to Good Clinical Practice guidelines, and adhere to the tenets of the declaration of Helsinki.

\subsection{Consent for publication $\{32\}$}

Please see appendices 2-6 for model consent form and other related documentation given to participants and authorised surrogates.

\subsection{Competing interests $\{28\}$}

The authors declare that they have no competing interests.

\section{References}


1. Chacra ZA, Manoukian JJ, Al-Qahtani K, Al-Eisa M, Balys R, Hagr A, et al. Hydrogen peroxide mouth rinse: an analgesic post-tonsillectomy. J Otolaryngol. 2005;34(3):178-82.

2. Dhiwakar M, Clement WA, Supriya M, McKerrow W. Antibiotics to reduce post-tonsillectomy morbidity. Cochrane database of systematic reviews (Online). 2012;12:CD005607.

3. Davidoss NH, Eikelboom R, Friedland PL, Santa Maria PL. Wound healing after tonsillectomy - a review of the literature. J Laryngol Otol. 2018;132(9):764-70.

4. Mann EA, Blair EA, Levy AJ, Chang A. Effect of topical antibiotic therapy on recovery after tonsillectomy in adults. Otolaryngology-head and neck surgery : official journal of American Academy of Otolaryngology-Head and Neck Surgery. 1999;121(3):277-82.

5. Colreavy MP, Nanan D, Benamer M, Donnelly M, Blaney AW, O'Dwyer TP, et al. Antibiotic prophylaxis post-tonsillectomy: is it of benefit? Int J Pediatr Otorhinolaryngol. 1999;50(1):15-22.

6. Miura MS, Saleh C, de Andrade M, Assmann M, Lima LH, Lubianca Neto JF. Topical clindamycin in post-adenotonsillectomy analgesia in children: a double-blind, randomized clinical trial. Otolaryngol Head Neck Surg. 2009;141(4):509-15.

7. Grandis JR VR, Rihs JD, Yu VL, Wagner RL, Kachman KK, Johnson JT. The efficacy of topical antibiotic prophylaxis for contaminated head and neck surgery. Laryngoscope.104(6):719-24.

8. Baradaranfar MH, Zand V, Dadgarnia MH, Atighechi S. Efficacy of clindamycin in reducing pain following tonsillectomy in adults: A double-blind, randomised trial. Journal of Laryngology and Otology. 2013;127(11):1106-10.

9. FAO/WHO. Report of a joint FAO/WHO expert consultation on guidelines for the evaluation of probiotics in food. World Health Organization and Food and Agriculture Organization of the United Nations, London Ontario, Canada. 2002.

10. Stamatova I, Meurman JH. Probiotics: health benefits in the mouth. Am J Dent. 2009;22(6):329-38.

11. Zhu B, Wang X, Li L. Human gut microbiome: the second genome of human body. Protein Cell. 2010;1(8):718-25.

12. Kasatpibal NW, J. D. Saokaew, S. Kengkla, K. Heitkemper, M. M. Apisarnthanarak, A. Effectiveness of Probiotic, Prebiotic, and Synbiotic Therapies in Reducing Postoperative Complications: A Systematic Review and Network Meta-analysis. Clin Infect Dis. 2017;64(suppl_2):S153-S60.

13. Skonieczna-Zydecka K, Kaczmarczyk M, Loniewski I, Lara LF, Koulaouzidis A, Misera A, et al. A Systematic Review, Meta-Analysis, and Meta-Regression Evaluating the Efficacy and Mechanisms of Action of Probiotics and Synbiotics in the Prevention of Surgical Site Infections and Surgery-Related Complications. J Clin Med. 2018;7(12).

14. Wescombe PA, Hale JD, Heng NC, Tagg JR. Developing oral probiotics from Streptococcus salivarius. Future Microbiol. 2012;7(12):1355-71.

15. Lima SF, Teixeira AG, Higgins CH, Lima FS, Bicalho RC. The upper respiratory tract microbiome and its potential role in bovine respiratory disease and otitis media. Sci Rep. 2016;6:29050. 
16. de Steenhuijsen Piters WA, Sanders EA, Bogaert D. The role of the local microbial ecosystem in respiratory health and disease. Philos Trans R Soc Lond B Biol Sci. 2015;370(1675).

17. Di Pierro F, Donato G, Fomia F, Adami T, Careddu D, Cassandro C, et al. Preliminary pediatric clinical evaluation of the oral probiotic Streptococcus salivarius K12 in preventing recurrent pharyngitis and/or tonsillitis caused by Streptococcus pyogenes and recurrent acute otitis media. Int J Gen Med. 2012;5:991-7.

18. Di Pierro F, Adami T, Rapacioli G, Giardini N, Streitberger C. Clinical evaluation of the oral probiotic Streptococcus salivarius $\mathrm{K} 12$ in the prevention of recurrent pharyngitis and/or tonsillitis caused by Streptococcus pyogenes in adults. Expert Opin Biol Ther. 2013;13(3):339-43.

19. Kaci G, Goudercourt D, Dennin V, Pot B, Dore J, Ehrlich SD, et al. Anti-inflammatory properties of Streptococcus salivarius, a commensal bacterium of the oral cavity and digestive tract. Appl Environ Microbiol. 2014;80(3):928-34.

20. Zupancic K, Kriksic V, Kovacevic I, Kovacevic D. Influence of Oral Probiotic Streptococcus salivarius K12 on Ear and Oral Cavity Health in Humans: Systematic Review. Probiotics Antimicrob Proteins. 2017;9(2):102-10.

21. Burton JP, Cowley S, Simon RR, McKinney J, Wescombe PA, Tagg JR. Evaluation of safety and human tolerance of the oral probiotic Streptococcus salivarius K12: a randomized, placebocontrolled, double-blind study. Food Chem Toxicol. 2011;49(9):2356-64.

22. Burton JP, Wescombe PA, Moore CJ, Chilcott CN, Tagg JR. Safety assessment of the oral cavity probiotic Streptococcus salivarius K12. Appl Environ Microbiol. 2006;72(4):3050-3.

23. Shishegar M, Ashraf MJ. Posttonsillectomy bacteremia and comparison of tonsillar surface and deep culture. Adv Prev Med. 2014;2014:161878-.

24. Tolska HK, Hamunen K, Takala A, Kontinen VK. Systematic review of analgesics and dexamethasone for post-tonsillectomy pain in adults. British journal of anaesthesia. 2019;123(2):e397-e411.

25. Diakos EA, Gallos ID, El-Shunnar S, Clarke M, Kazi R, Mehanna H. Dexamethasone reduces pain, vomiting and overall complications following tonsillectomy in adults: a systematic review and metaanalysis of randomised controlled trials. Clinical otolaryngology : official journal of ENT-UK ; official journal of Netherlands Society for Oto-Rhino-Laryngology \& Cervico-Facial Surgery. 2011;36(6):53142.

26. Haefeli M, Elfering A. Pain assessment. Eur Spine J. 2006;15 Suppl 1(Suppl 1):S17-S24.

27. Ng TT, Diamantaras D, Priestley J, Redman J, De Silva N, Mahanta V. Is celecoxib a useful adjunct in the treatment of post-tonsillectomy pain in the adult population? A randomised, double-blind, placebo-controlled study. The Journal of laryngology and otology. 2017;131(S1):S18-S28.

28. Murdoch Children's Research Institute (MCRI). Research Resources: Clinical Trial (Drug/Device) Protocol template [Internet]. Melbourne Children's Trial Centre, Melbourne, Australia: Murdoch Children's Research Institute (MCRI) Clinical Research Development Office (CRDO); 2019 [Available from: https://www.mcri.edu.au/research/training-and-resources/clinical-research-development-officecrdo/resources-quantitative. 
29. NMHRC Council. Guidance: Safety monitoring and reporting in clinical trials involving therapeutic goods. Nov 2016.

\section{Figures}

\begin{tabular}{|c|c|}
\hline & $\begin{array}{l}\text { Adult patients awaiting tonsillectomy will be identified from } \\
\qquad \text { Hospital waiting list }\end{array}$ \\
\hline & 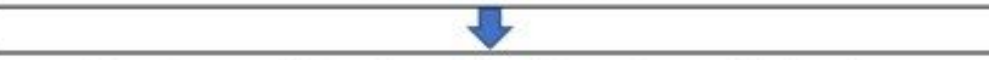 \\
\hline & $\begin{array}{l}30 \text { patients will be selected and have letter of invitation to } \\
\text { participate and PICF sent to them via mail or email }\end{array}$ \\
\hline & $\checkmark$ \\
\hline \multirow{19}{*}{$\begin{array}{l}\text { Excluded: Not } \\
\text { eligible to } \\
\text { participate }\end{array}$} & Patients will be contacted via telephone (using telephone script) to \\
\hline & exclusion criteria \\
\hline & Y \\
\hline & $\begin{array}{l}\text { Patient interested in participating will be seen on day of surgery by } \\
\text { delegated member and a PICF will be signed to obtain consent for } \\
\text { trial participation }\end{array}$ \\
\hline & V \\
\hline & $\begin{array}{c}\text { Patients will be randomly assigned via block randomisation to } \\
\text { placebo or probiotic group (Hospital pharmacist) }\end{array}$ \\
\hline & $\sqrt{2}$ \\
\hline & $\begin{array}{l}\text { All patients will receive standard general anaesthetic and have all } \\
\text { medications listed under section of concomitant therapy }\end{array}$ \\
\hline & $\begin{array}{l}\text { Postoperatively the patients will receive probiotic or placebo in a } \\
\text { sealed plastic bag on discharge by hospital pharmacist. Instructions } \\
\text { on how to use probiotic and analgesia will be provided }\end{array}$ \\
\hline & $\sqrt{2}$ \\
\hline & $\begin{array}{l}\text { All patients will go home day } 1 \text { postoperatively (electronic } \\
\text { questionnaire will be sent to patient via email or mobile) }\end{array}$ \\
\hline & र \\
\hline & $\begin{array}{l}\text { Day } 1 \text { patients will have instructions of how to use gargles and } \\
\text { complete questionnaire }\end{array}$ \\
\hline & $\sqrt{2}$ \\
\hline & Day 5-7 follow up in outpatients/telephone \\
\hline & 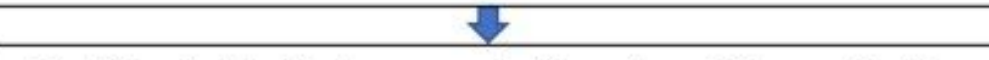 \\
\hline & Day 14 outpatient follow up; collection of remaining medications \\
\hline & $\sqrt{2}$ \\
\hline & Day 28 follow up in outpatients \\
\hline
\end{tabular}

Figure 1 
Flow chart of trial process from allocation to follow-up. Peninsula Health

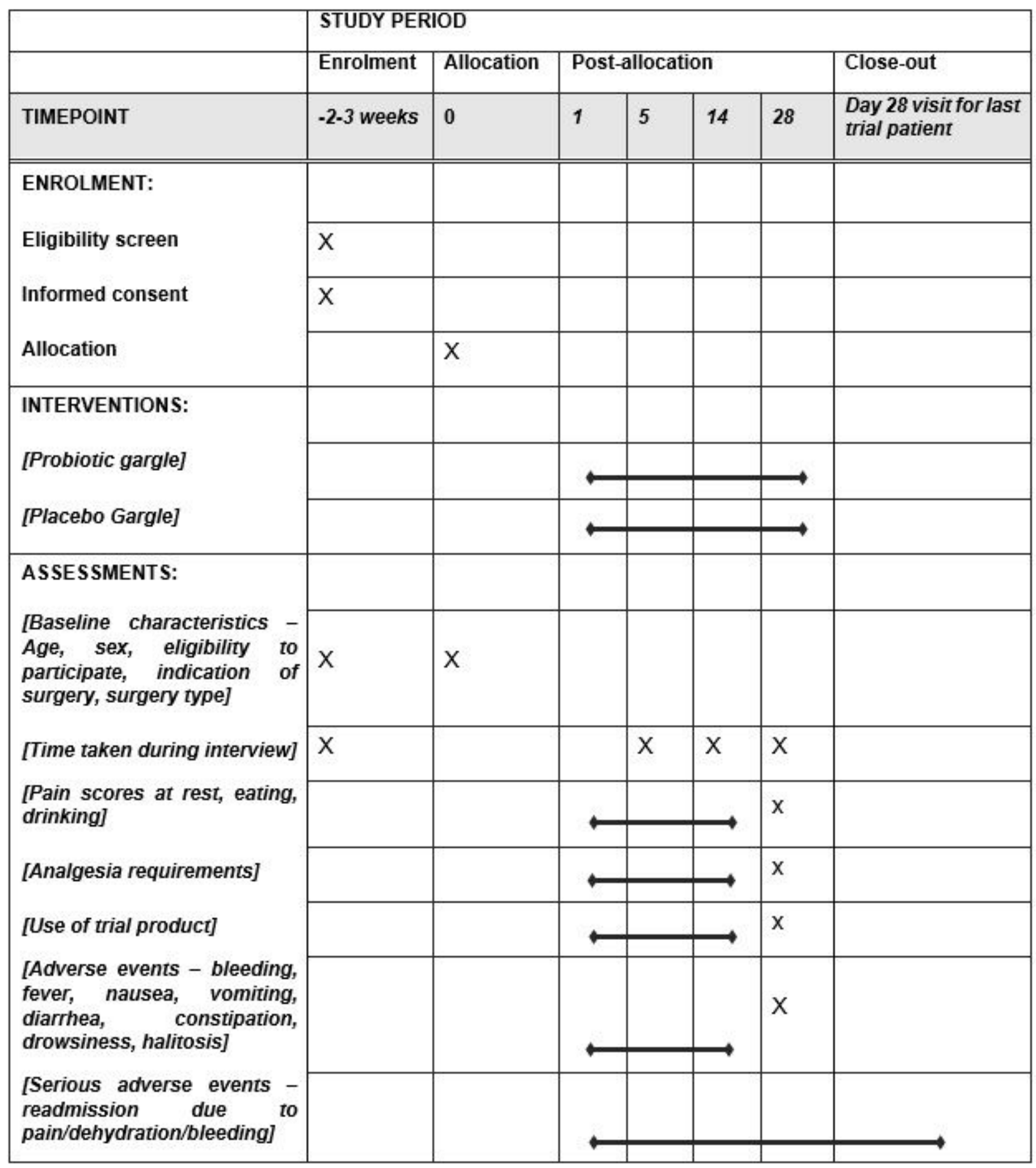

Figure 2

Standard Protocol items: Recommendations for Interventional Trials (SPIRIT) figure of trial schedule for enrolment, interventions and follow-up assessments. Peninsula Health *Recommended content can be displayed using various schematic formats. See SPIRIT 2013 Explanation and Elaboration for examples from protocols. ** List specific timepoints in this row. 


\begin{tabular}{|l|l|l|l|l|l|l|}
\hline Adverse Event & \multicolumn{2}{l|}{ Probiotic $(\mathbf{N}=\mathbf{2 7})$} & \multicolumn{3}{l|}{ Placebo $(\mathbf{N}=\mathbf{2 9})$} \\
\hline Intensity & Mild & Moderate & Severe & Mild & Moderate & Severe \\
\hline Total & 13 & 1 & 0 & 13 & 6 & 2 \\
\hline
\end{tabular}

\section{Figure 3}

Summary of adverse events by intensity. Adapted from Burton JP et al (23)

\section{Supplementary Files}

This is a list of supplementary files associated with this preprint. Click to download.

- Appendix1LetterlnvitingParticipation.docx

- Appendix2PatientInformationandConsentFormPICF.docx

- Appendix3ElectronicsurveyDaily14dayquestionnaire.docx

- Appendix4PatientInstructionsanddiarysheet.docx

- Appendix5Telephonescriptforday5review.docx

- Appendix6SignificantSafetylssuesSSI.docx

- Appendix7ExpeditedSafetyReportForm.docx

- Appendix8Ethicsapprovalletter.pdf 